\title{
LIMIT DEFORMATION ANALYSIS OF UNSATURATED EXPANSIVE SOILS DURING WETTING AND DRYING CYCLES
}

\author{
Kai Li ${ }^{1,2}$, H. Nowamooz ${ }^{2}$, C. Chazallon', B. Migault ${ }^{2}$ \\ ${ }^{1}$ Qingdao Key Laboratory for Geomechanics and Offshore Underground Engineering, \\ Qingdao University of Technology, Qingdao, China; ${ }^{2}$ Group of Civil Engineering in \\ Laboratory of ICUBE, University of Strasbourg, Strasbourg, France.
}

UDC 624.151 .5

\begin{abstract}
Experimental observation on unsaturated expansive soils subjected to wetting and drying cycles presents a stabilized limit equilibrium state at the end of the suction cycles. Models developed previously for this behavior introduce a large number of parameters which lead to a time-consuming procedure to calibrate. This paper develops a limit analysis method with the Zarka concept for the mechanical behavior of expansive soils subjected to wetting and drying cycles. The required parameters of the proposed limit analysis method are calibrated by the experimental results obtained for bentonite/sand mixtures subjected to cyclic suction loadings in an odometric test. The comparison between the test results and the model predictions demonstrates the capacity of the proposed method to calculate the limit deformation during suction cycles and simulate the subsequent mechanical behavior of the expansive soils after suction cycles.
\end{abstract}

\section{Introduction}

Unsaturated expansive soil is susceptible to the effect of environmental conditions because an increase in water content causes the soil to swell, while water removal causes the shrinkage of soil. Many engineered structures built on this material are significantly damaged and cracked, such as shallow foundations, drainage channels, and buffers of radioactive waste disposal. Determination of possible swell and settlement of expansive soils due to suction changes are generally considered as a necessary part of the construction design. Furthermore, the hydromechanical response of expansive soils affected by wetting and drying cycles is essential information for understanding its constitutive behavior. Laboratory tests on these materials during wetting and drying cycles reported by several researchers [1-4] show that a limit equilibrium state can be reached after several wetting and drying cycles. In this context, the limit deformation at the equilibrium state after wetting and drying cycles should be studied to better understand the hydromechanical behavior of unsaturated expansive soils.

Many models were proposed to predict the complex hydromechanical behavior of unsaturated expansive soils with the application of suction cycles [5-8]. The widely accepted model is the Barcelona expansive model (BExM) developed by Alonso et al. [2, 3, 8], able to simulate the basic behavior of unsaturated expansive soil, including the strain fatigue phenomenon during wetting-drying cycles and the prediction of the final equilibrium state at the end of the suction cycles. However, this model presents a large number of parameters such as the coupling functions for micro- and macrostructural strains. The calibration of these parameters needs several experimental tests, which lead to a time-consuming procedure to characterize their mechanical behavior.

Translated from Osnovaniya, Fundamenty i Mekhanika Gruntov, No. 1, p. 32, January-February, 2018. 


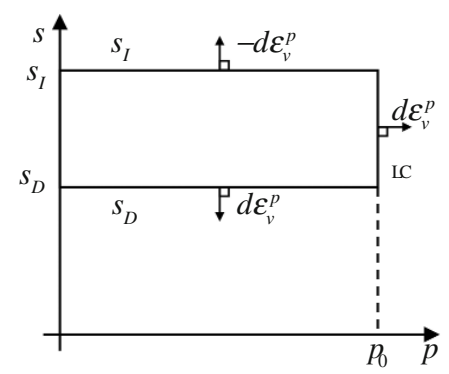

Fig.1. Yield surfaces.

The Zarka limit analysis method is a simplified theory, initially developed for kinematic hardening materials, such as metals $[9,10]$. It defines the accumulated plastic strains at limit equilibrium states with Melan's static theorem by introducing less model parameters. Sharp et al. [11] applied a similar limit analysis concept for the first time to the unbound granular materials, which defined the limit load as the key design load. Chazallon et al. [12, 13] have developed an elastoplastic model based on the Zarka limit state theory with a nonassociated flow rule for unbound granular materials when they are subjected to repeated load triaxial tests in order to determine the limit accumulation of plastic strains.

This paper presents a limit approach based on the Zarka analysis method to model the mechanical behavior of unsaturated expansive soils subjected to wetting and drying cycles. The required parameters of the proposed method are calibrated by the odometric test results carried out on different initial states of an expansive soil. The model is validated for the intermediate samples to compare model calculations with experimental results. These comparisons show that the proposed model is able to replace the step-by-step method to estimate limit accumulated plastic strains of expansive soils during suction cycles and the subsequent mechanical behavior after suction cycles.

\section{Simplified yield surfaces for limit analysis}

A simplified yield surface is employed in the Zarka limit analysis to simulate the mechanical behavior of unsaturated expansive soils during the successive wetting and drying cycles.

It is generally accepted that a unique plane (net mean stress-suction) is sufficient to describe the mechanical behavior of unsaturated soils. Figure 1 shows a rectangular yield surface in the net mean stresssuction plane representing the elastic domain. The equations of the different boundaries can be given by

$$
\begin{aligned}
& s=s_{I}, \\
& s=s_{D}, \\
& p=p_{0},
\end{aligned}
$$

where, $s_{I}$ the suction increase limit, $s_{D}$ the suction decrease limit, and $p_{0}$ is the preconsolidation stress.

We take into account the following hypotheses for the yield surfaces: 1) the width of the elastic domain is small, not changing with suction cycles. The suction variation within the rectangular will result in the elastic volumetric strain:

$$
d \varepsilon_{v s}^{e}=\frac{\kappa_{s}}{v} \frac{d s}{s},
$$

where $v$ is the specific volume, equal to $1+e_{0}$, where $e_{0}$ is the initial void ratio of the sample; 2) the length of the rectangular depends on the suction level. Based on this hypothesis, we take advantage of the loading collapse (LC) curve defined in the BBM model [14]. The relation between the preconsolidation stress and suction level can be given by

$$
p_{0}(s)=p_{c}\left(\frac{p_{0}^{*}}{p_{c}}\right)^{\frac{\lambda(0)-\kappa}{\lambda(s)-\kappa}},
$$




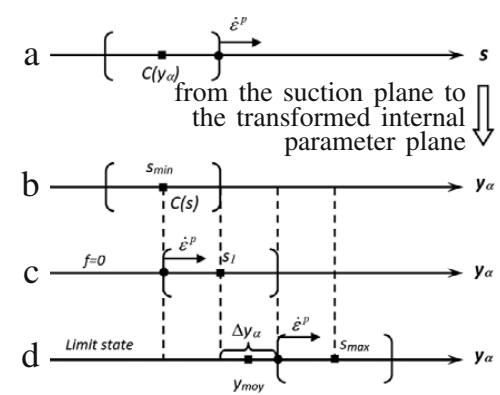

Fig.2. Transformed internal parameter plane for the limit analysis.

where $p_{0}^{*}$ is the saturated preconsolidation stress, $p_{c}$ is a reference stress, and $\lambda(0)$ is the elastic-plastic stiffness index for loading variation at the saturated state.

The increase of net mean stress within the rectangular will generate the elastic volumetric strain:

$$
d \varepsilon_{v p}^{e}=\frac{\kappa}{v} \frac{d p}{p}
$$

and if the boundary $p=p_{0}(s)$ is reached, the following total volumetric strain will be given by

$$
d \varepsilon_{v p}=\frac{\lambda(s)}{v} \frac{d p_{0}}{p_{0}},
$$

in which $\kappa$ and $\lambda(s)$ are the elastic stiffness index and the elastic-plastic stiffness index for loading variation, respectively.

The above simplified yield surfaces for the isotropic stress state is only used in the limit deformation analysis in the following section, instead of step-by-step calculation of material deformations, although it has a similar formulation as the BBM or BExM model.

\section{Limit deformation analysis with the Zarka concept}

The Zarka limit analysis is based on an associated elastic-plastic model (von Mises yield surface) with linear kinematic hardening. For this simplified method, Zarka introduces a series of transformed internal variables to characterize the mechanical system; then a local geometrical construction in the transformed internal parameter plane is performed to estimate the stabilized limit state and the associated deformation components.

The proposed yield surfaces of suction variations with a linear kinematic hardening can be written as

$$
f=\left|s-y_{\alpha}\right|-s_{\alpha},
$$

where $s_{\alpha}$ is the threshold value of elastic limit for suction variation and $y_{\alpha}$ is the kinematic hardening variable, which can be related to the plastic strain,

$$
y_{\alpha}=h \varepsilon^{p},
$$

where $h$ is the kinematic hardening modulus.

When $f=0$, the yield surface equation can be written as

$$
s=s_{\alpha}+y_{\alpha} \text {. }
$$

In the suction plane, the normality law of plasticity can be written with Moreau's notation:

$$
\dot{\varepsilon}^{p} \in \partial \phi_{C\left(y_{\alpha}\right)}(s) \text { with } s \in C\left(y_{\alpha}\right) \text {, }
$$

where $\partial \phi_{C\left(y_{\alpha}\right)}(s)$ is the subdifferential to the convex $C\left(y_{\alpha}\right)$, where the plastic strain rate is an external normal to the convex $C\left(y_{\alpha}\right)$ (Fig.2a). 
We present the convex in the transformed internal parameter $y_{\alpha}$ plane (Fig.2b) and rewrite the yield surface when $f=0 ; y_{\alpha}$ can be expressed in terms of the given applied stress,

$$
y_{\alpha}=s-s_{\alpha} \text {. }
$$

where $y_{\alpha}$ represents the transformed internal parameter; this equation indicates the yield surface centered in the applied suction $s$ with radius $s_{\alpha}$ that translates in the transformed internal parameter $y_{\alpha}$ plane. In this transformed internal parameter $y_{\alpha}$ plane, the normality law of plasticity becomes

$$
\dot{\varepsilon}^{p} \in-\partial \phi_{C(s)}\left(y_{\alpha}\right) \text { with } y_{\alpha} \in C(s) \text {, }
$$

where $-\partial \phi_{C(\mathrm{~s})}\left(y_{\alpha}\right)$ is the subdifferential to the convex $C(s)$, where the plastic strain rate is an internal normal to the plasticity convex (Fig.2c).

When the suction amplitude is large, the extreme position of the convex translates in the transformed internal parameter plane with $s_{\max }$ (Fig.2d). In this case, the limit equilibrium state is reached, and the limit deformations during the suction cycles between $s_{\min }$ and $s_{\max }$ can be calculated by the limit analysis in the following section.

To perform the limit analysis, the proposed yield surface is presented in the transformed internal parameter $y_{\alpha}$ plane. In this transformed internal parameter plane, the convex that characterizes the behavior of the soil sample translates between suction $s_{\min }$ and suction $s_{\max }$ during the wetting and drying cycles.

Based on the Zarka limit analysis method, the variation of the volumetric plastic deformation during suction cycles can be computed by

$$
\Delta \varepsilon_{v s}^{p}=\frac{1}{h} \Delta y_{\alpha}
$$

where $y_{\alpha}$ can be determined in the transformed internal parameter plane when the minimum suction and the maximum suction are given.

With the application of wetting and drying cycles, the limit equilibrium state can be obtained at the end of the suction cycles where no additional plastic strain accumulation can be observed. Because we have far too few experimental points at the equilibrium state, the hysteresis phenomenon cannot be described precisely. Consequently, a linear variation of the elastic strain with the suction is supposed at the limit equilibrium state.

It can be written as

$$
\Delta \varepsilon_{v s}^{e}=\frac{\kappa_{s}}{v} \frac{\Delta s}{s}
$$

where $\kappa_{s}$ is the elastic stiffness index for suction variation.

Gens et al. [2] stated that the fatigue of swelling associated with suction cycles under a vertical pressure is due to the macrostructural softening induced by microstructural expansion. In this case, the loading collapse yield surface presented in the net mean stress-suction plane shifts to the left of the initial LC yield surface. On the other hand, an increase in shrinkage deformation causes the shifting of the loading collapse yield surface to the right of the initial LC yield surface accompanied by macrostructural hardening behavior.

Experimental evidence shows a strong dependence of the preconsolidation stress on the macrostructural plastic strain during wetting and drying cycles. For simplicity, we assume that the increment of preconsolidation stress is proportional to the variation of volumetric plastic strain due to suction cycles, the same equation as the BExM:

$$
\frac{d p_{0}^{*}}{p_{0}^{*}}=\frac{1+e_{0}}{\lambda(0)-\kappa} d \varepsilon_{v s}^{p} .
$$

Based on this equation, the volumetric plastic deformation estimated by the proposed limit analysis can be used to determine the preconsolidation stress for the saturated state $p_{0}^{*}$. Thereafter, this saturated preconsolidation stress $p_{0}^{*}$ is used to find the preconsolidation stress $p_{0}(s)$ as well as the final position of the LC yield surface through Eq.(5). Finally, the subsequent mechanical behavior of expansive soils after wetting and drying cycles can be simulated. 
TABLE 1

\begin{tabular}{|c|c|c|c|c|c|c|}
\hline Parameters & \multicolumn{3}{|c|}{ Loose samples } & \multicolumn{3}{c|}{ Dense samples } \\
\hline Net mean stress, kPa & 15 & 30 & 60 & 15 & 30 & 60 \\
\hline $\begin{array}{c}\text { Hardening modulus } \\
h, \mathrm{MPa}\end{array}$ & 126 & 79 & 60 & -125 & -163 & -540 \\
\hline $\begin{array}{c}\text { Elastic region limit } \\
s_{I}-s_{D}, \mathrm{MPa}\end{array}$ & 0 & 0 & 0 & 0 & 0 & 0 \\
\hline $\begin{array}{c}\text { Elastic stiffness index } \\
\text { for suction variation } \kappa_{s}\end{array}$ & 0.02 & 0.02 & 0.02 & 0.04 & 0.04 & 0.04 \\
\hline
\end{tabular}

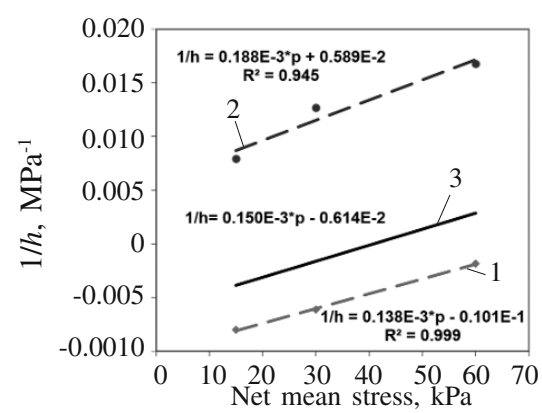

Fig.3. Evolution law of the inverse of the hardening modulus with net mean stress for different samples: ) calibrated value (D); -) calibrated value (L); 1) linear law (D); 2) linear law (L); 3) linear prediction (I).

\section{Validation of the proposed method}

Nowamooz et al. $[1,4,15]$ studied the mechanical behavior of an artificially prepared mixture of $40 \%$ silt and $60 \%$ bentonite after several drying and wetting cycles. The samples were compacted with the same initial water content $(w)$ of $15 \%$. The three different initial dry densities $\left(\gamma_{d}\right)$ were $1.27,1.48$, and $1.55 \mathrm{Mg} \cdot \mathrm{m}^{-3}$, corresponding to loose (L), intermediate (I), and dense (D) soils, respectively.

From experimental results, the hardening module $h$ can be deduced through Eq.(14) since we know the accumulated plastic deformation as well as the transformed internal parameter at a given net mean stress. The elastic stiffness index for suction variation $\kappa_{s}$ can be estimated by Eq.(15) from the last wetting and drying cycle at the limit equilibrium state. Table 1 summarize the required parameters: hardening modulus $(h)$, elastic stiffness index for suction variation $\left(\kappa_{s}\right)$, and elastic region limit $\left(s_{I}-s_{D}\right)$ of the proposed method for loose and dense samples, respectively. Comparing the accumulated plastic strain during suction cycles, the elastic strain is very small, and we assume $s_{I}-s_{D}$ are equal to zero for the sake of simplification.

Because of the volumetric shrinkage strains during the suction cycles, a positive sign was used for the $h$ values to show the hardening phenomenon. The inverse of the hardening modulus $(1 / h)$ varies linearly with net mean stress. Figure 3 shows the evolution law of the inverse of $1 / h$ with the net mean stress $p$ for loose and dense samples, respectively. The accumulated plastic deformation increases with increase in net mean stress.

We can propose the following linear expression between $1 / h$ and $p$

$$
1 / h=A p+B,
$$

where $A$ and $B$ are material constants.

Figures $4 \mathrm{a}$ and $4 \mathrm{~b}$ present the model calibration compared with experimental results at different net mean stress for the loose and dense samples, respectively. The model estimations show the equilibrium state reached for both loose and dense materials where they exhibit a reversible behavior at the end of the drying and wetting cycles, and the model calculations produce a good agreement with small relative errors. 

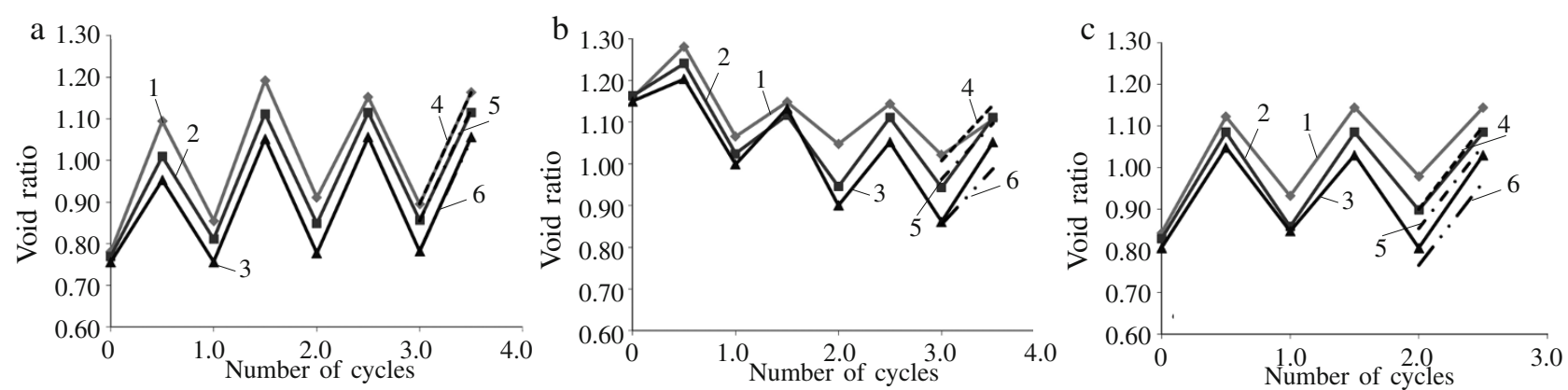

Fig.4. Comparison of test results with model predictions for: a) loose samples; b) dense samples;

c) intermediate samples at different net mean stresses: 1) test $(15 \mathrm{kPa})$; 2) test $(30 \mathrm{kPa})$;

$3)$ test $(60 \mathrm{kPa})$; 4) model $(15 \mathrm{kPa})$; 5) model $(30 \mathrm{kPa})$; 6) model $(60 \mathrm{kPa})$.

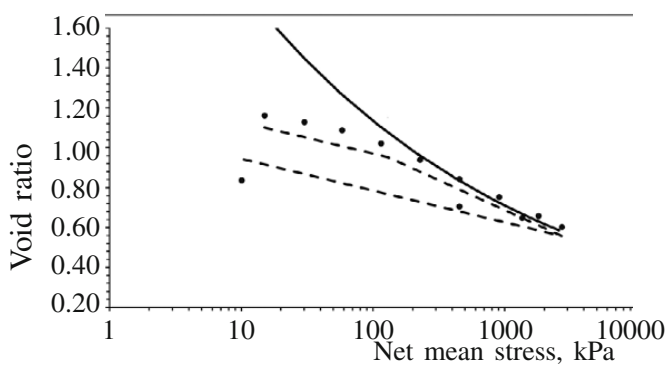

Fig.5. Comparison of loading-unloading compression test results with model predictions for intermediate samples at the saturated state: •) loading-unloading compression tests after wetting and drying; ----) model prediction of loading-unloading compression tests; $\longrightarrow$ normally consolidated curve.

The validation of the model is carried out with tests of the intermediate samples. The model parameter for the intermediate samples $\left(s_{I}-s_{D}\right)$ is also taken equal to zero, the same as the loose and dense states. The parameters $A$ and $B$ for the intermediate samples $\left(\gamma_{d}=1.48 \mathrm{Mg} / \mathrm{m}^{3}\right)$ based on the previous estimated values of the loose and dense samples are: $A=0.150 \mathrm{MPa}^{-2} ; B=-0.614 \mathrm{E}-02 \mathrm{MPa}^{-1} ; \kappa_{s}=0.03$. As to the parameter, the elastic stiffness index for $\kappa_{s}$ for intermediate sample also can be interpolated by the values of the loose and dense samples. Additionally, the extrapolated line of the inverse of the hardening modulus $(1 / h)$ for intermediate samples is illustrated in Fig.3.

On the basis of the predicted linear law of kinematic hardening modulus, Fig. $4 \mathrm{c}$ presents a comparison of test results with the model predictions for intermediate soil at different net mean stress. For intermediate soils, the initial state is closer to the reversible line, and these soils needs less suction cycles to obtain the equilibrium state. The relative error varies between $5.1 \%$ and $8 \%$, confirming the capacity of the model to predict the accumulated plastic strains for another initial state.

The coupling behavior between suction cycles and mechanical properties is simulated in this section, and the following values of soil constants are used in this simulation: $\lambda(0)=0.136, \kappa=0.069, p_{0}^{*}=130 \mathrm{kPa}$. In this study, only the simulations for the intermediate samples are presented, and the same procedures can be conducted for both loose samples and dense samples.

Since the limit deformation during wetting and drying cycles can be given through the proposed limit analysis method in the previous section, the preconsolidation stress at the saturated state can be calculated by Eq.(16) and the coupling behavior between suction cycles and mechanical properties can be simulated on the basis of the given model parameters.

Because the intermediate initial state is close the stabilized limit equilibrium state, model simulations show a small softening mechanical behavior of the studied material. Figure 5 shows a comparison of the model prediction with the experimental results for the intermediate samples. In this figure, the 
compression curves with the application of suction cycles converge to the normally consolidated curve (NCC) and model predictions provide a good qualitative match to the experimentally observed behavior.

\section{Conclusion}

In this work, the limit analysis with a linear kinematic hardening is performed to simulate the mechanical behavior of expansive soils subjected to wetting and drying cycles. The limit deformation during suction cycles can be investigated in the transformed internal parameter plane with a rectangular shape for the yield surface. For the proposed limit analysis of unsaturated expansive soils during suction cycles, we emphasize that the parameter $1 / h$, which depend on $A$ and $B$, and the parameters $\kappa_{s}$ and $\left(s_{I}-s_{D}\right)$ are sufficient to determine the final limit state. It is important to determine this limit equilibrium state because it presents the final mechanical behavior of unsaturated expansive soil subjected to several suction cycles. The calculated limit deformation during suction cycles can be employed in the coupling law to show the influence of suction cycles on the subsequent mechanical behavior. The same model parameters and calibration procedures as the BExM model can be used and a good qualitative match with the experimental results has been obtained.

\section{Acknowledgments}

This work is financially supported by the projects of the Natural Science Foundation of Shandong Province (Grant No: ZR2016AB18) and the National Natural Science Foundation of China (Grant No: 11572165).

\section{REFERENCES}

1. H. Nowamooz and F. Masrouri, "Hydromechanical behavior of an expansive bentonite/silt mixture in cyclic suction-controlled drying and wetting tests," Eng. Geol., 101, 154-164 (2008).

2. A. Gens and E. E. Alonso, "Framework for the behavior of unsaturated expansive clays," Can. Geotech. J., 29, 1013-1032 (1992).

3. E. E. Alonso, E. Romero, C. Hoffmann, and E. G. Escudero, "Expansive bentonite/sand mixture in cyclic controlled suction drying and wetting," Eng. Geol., 81, 213-226 (2005).

4. H. Nowamooz, E. Jahangir, and F. Masrouri, "Volume change behavior of a swelling soil compacted at different initial states," Eng. Geol., 153, 25-34 (2013).

5. Y. J. Cui, M. Yahia-aissa, and P. Delage, "A model for the volume change behavior of heavily compacted swelling clays," Eng. Geol., 64, 233-250 (2002).

6. S. J. Wheeler, R. S. Sharma, and M. S. R. Buisson, "Coupling of hydraulic hysteresis and stressstrain behavior in unsaturated soils," Geotechnique, 53, 41-54 (2003).

7. W. J. Sun and D. A. Sun, "Coupled modelling of hydro-mechanical behavior of unsaturated compacted expansive soils," Int. Numer. Anal. Methods Geomech., 36, 1002-1022 (2011).

8. E. E. Alonso, J. Vaunat, and A. Gens, "Modelling the mechanical behavior of expansive clays," Eng. Geol., 54, 173-183 (1999).

9. J. Zarka and J. Casier, "Elastic plastic response of structure to cyclic loading: practical rules," Mechanics Today, Pergamon Press, Oxford, 93-198 (1979).

10. J. Zarka, J. Frelat, G. Inglebert, and P. Kasmai-Navid, A New Approach in Inelastic Analysis of Structures, M. Nijhoff, Dordrecht (1990).

11. R. Sharp and J. Booker, "Shakedown of pavements under moving surface load," J. Transp. Eng., 110, 1-14 (1984).

12. C. Chazallon, G. Koval, P. Hornych, F. Allou, and S. Mouhoubi, "Modelling of rutting of two flexible pavements with the shakedown theory and the finite element method," Comput. Geotech., 36, 798-809 (2009).

13. C. Chazallon, F. Allou, P. Hornych, and S. Mouhoubi, "Finite element modelling of the long term behavior of a full scale flexible pavement with the shakedown theory," Int. Numer. Anal. Methods Geomech., 33, 45-70 (2009).

14. E. E. Alonso, A. Gens, and A. Josa, "A constitutive model for partially saturated soils," Geotechnique, 40, 405-430 (1990).

15. H. Nowamooz and F. Masrouri, "Mechanical behavior of expansive soils after several drying and wetting cycles," Geomech. Geoeng., 5, 213-221 (2010). 